\title{
Performance Assurance for DC Cables for Electric Ships
}

\author{
Robert E. Hebner, Angelo L. Gattozzi \\ University of Texas at Austin \\ Austin, TX, USA
}

\begin{abstract}
Minimizing the weight and volume in a ship cable plant improves system efficiency or provides the capability to carry more critical payloads. Cabling should be as small as possible. This research developed an approach to the fundamental understanding needed to design, specify, and test cabling that has the minimum prudent size with high reliability. Progress requires a convolution of the failure modes with the thermal, electrical, and mechanical environment in which the cable is operating.
\end{abstract}

Index Terms-Breakdown, dc cable, electric ship, faults, insulation, microgrid

\section{INTRODUCTION}

If ships were not volume and weight constrained, the selection of cabling for a dc ship would be straightforward. The designer would simply pick a cable topology that works well in a land-based system and select a voltage rating one or two steps above the ships operating voltage to compensate for unknown differences between the land and ship environments. In reality, there are two major problems with this direct approach. First, the experience base with dc cables in the 10$20 \mathrm{kV}$ range is quite limited. Second, ships are extremely weight and space constrained. Moreover, an overdesigned cabling system is a penalty to ship performance for the life of the ship.

Conventional cable design and/or selection typically balance three interrelated factors to achieve a robust design: thermal behavior, the transient environment, and longer-term degradation. At the most fundamental level, the cable conductor size must be selected so that the resistive heating of the cable [1] does not stress excessively the thermal ratings of the cable across the range of operating currents and ambient temperatures. The transient environment is typically the environment that initiates cable failure. While continuous conditions may slowly degrade a cable's withstand voltage, transients are usually what exceeds the degraded level. Longterm degradation is manifested as slow charge migration [2] or changes in the partial discharge spectrum $[3,4]$.

In an effort to determine the cable system's reliability, this research is exploring how small and light a cable can be for a ship environment. The key attributes of the environment that are examined include the thermal environment, the electrical use environment, and the required lifetime. The assessment focuses on polymeric insulation, whether in the form of the plastic material used in cables or the epoxy used in bus pipe or

\author{
Steven D. Pekarek \\ Purdue University \\ West Lafayette, IN, USA
}

bus bar systems. This is not an effort to select among the existing available cable options but to explore the trade space available for future electric ships.

The three key features of this approach are:

1. Use modeling and simulation to anticipate the electrical environment and to determine the degree that the electrical environment can be controlled at higher voltages. The Navy has much more control over its power system than does the conventional electrical utility.

2. Exploit the fact that the possible fundamental failure mechanisms have been identified in support of conventional utilities. The key factors are nonelectrical, i.e., thermal, mechanical, and chemical, residual voids from manufacturing, electrically induced voids, i.e. treeing, and space charge injection. So, the research focuses on which mechanisms are expected to be dominant in the specific environment, not on finding new mechanisms.

3. Use a combination of ac ship experience, focused experiments, and modeling to significantly reduce the time and cost of field testing.

\section{THERMAL CONSIDERATIONS}

The cables are assumed to operate at ambient temperatures and are composed of, at least, a metallic conductor and one of a broad range of polymeric materials to separate the conductor from the remainder of the environment. This can be a conventional cable, a bus pipe, or other configurations, but will be generically referred to as cable in this work to simplify the description.

The conductor size is set by the current in the cable and the insulation thickness is determined by the voltage. The material's properties couple the analysis, so the designs are not independent. The conventional approach to cable thermal modeling is the Neher-McGrath approach [1]. For a co-axial cable that approach, in a simple system, yields a model of the form:

$$
\mathrm{I}=\left[\left(\mathrm{T}_{\mathrm{c}}-\mathrm{T}_{\mathrm{a}}\right) /\left(\mathrm{RR}_{\mathrm{i}}\right)\right]^{1 / 2}
$$

where $I$ is the allowable current in the conductor, $T_{c}$ is temperature in the conductor due to resistive losses, $\mathrm{T}_{\mathrm{a}}$ is the ambient temperature, $\mathrm{R}$ is the resistance per unit length of the

This work was sponsored in part by the Office of Naval Research 
conductor and $\mathrm{R}_{\mathrm{i}}$ is the thermal resistance of the electrical insulation.

The glass transition temperature of most of the polymeric insulating materials used is about $90^{\circ} \mathrm{C}$. So, $\mathrm{T}_{\mathrm{c}}-\mathrm{T}_{\mathrm{a}}$ should not exceed $50^{\circ} \mathrm{C}$ under anticipated operating conditions, as $\mathrm{T}_{\mathrm{a}}$ can be in excess of $35^{\circ} \mathrm{C}$. If a larger temperature difference is needed, it limits the classes of available materials.

Although all events over the rated operating temperature of a cable degrade life, most cables operate at elevated temperature for part of their life due to unforeseen activities in the power system. Earlier work has shown that, for controlled thermal aging tests, a precipitous drop in electrical performance did not occur until the over temperature condition exceeded hundreds of hours [5]. This is consistent with field experience showing that temporally constrained occasional excursions over the rated temperature can be well tolerated.

This behavior is interesting in the context of cabling that is expected to have intermittent use on ships. For example, electromagnetic guns and high-powered lasers require significant current for operation. The current, however, is in the form of pulses and the systems are used intermittently. This leads to a condition in which careful measurements should be made on the specific insulation system to determine the minimum conductor size that produces an acceptable thermal transient to maintain the life of the cables for decades. Wasted weight and space would be necessary if these cables were specified for continuous duty.

\section{ELECTRICAL CONSIDERATIONS}

The electrical considerations make it attractive to consider that the cable can be made smaller. The maximum breakdown strength reported in polymeric insulation is around $10 \pm 5 \mathrm{MV} / \mathrm{cm}$, where the variation is due to the different polymers tested [6]. Consequently, if this intrinsic strength could be realized, the insulation system could be film $0.002 \mathrm{~mm}$ thick, making the cable size virtually equal to the conductor size.

Practical considerations make that impossible, but it would be equally naive to assume that the considerations that lead to the cable thicknesses commercially available today are representative of the ship environment. The way that the scaling is done is critical. Generically, insulations systems are designed in three steps [7]. The most basic step is the basic materials properties selection. This requires assessing the materials that appear to generally have appropriate dielectric strength, glass transition temperature, mechanical properties, and aging properties for the application. The second step is to determine through experience, past records, and/or model tests the practical design stress that can be achieved. In cables, an explicit area effect is often also considered. Since failure is a weak-link phenomenon, the probability of stressing a lower failure voltage portion of the insulator increases as the area increases. Finally, one must assess the performance in the particular transient environment.

For cables, like most power system components, the highest design stress [7] is the lightning breakdown voltage [8]. The standard lightning impulse is a reasonable test waveform for apparatus to be attached to the terrestrial grid. Experience has shown that the waveform does not do undue harm to devices under tests and designs that withstand this test generally withstand the lightning-induced transients on the terrestrial power grid. It should be noted that the waveform is not based exclusively on the physics of a lightning strike. Rather, it is based on the average observed waveforms at a point in the grid when lightning struck at or near another point - it is an undefined convolution of the properties of the lightning and the grid. So, the highest design voltage for conventional cables is due to a phenomenon that does not exist in the same form in ships. The recent open literature studies of lightning strikes on ships focus on radiated electromagnetic effects rather than direct conducted effects on the ship itself [9]. The conducted effects determine whether this mechanism is responsible for the highest voltage seen by the power system or if there are other more significant voltages.

Lightning strikes on ships are rare [9]. As ships evolve in a way that more structural members are made of composites rather than metal, it can be expected that the effects of these rare strikes will change. As the metallic shielding decreases, the probability of coupling some of the energy in the lightning event to the ship power system may increase. This is an issue that the aircraft industry has been addressing for more than thirty years [10] and the changing composition of the fuselage of more modern planes compared to legacy aircraft means work continues.

An additional consideration is the system to provide shore power. Shore power connects the ship power system to the terrestrial grid. If it is possible to reduce the ship insulation size relative to that dictated by prudent practice for landbased systems, the two systems would interconnect without risk for the nominal power connections. Additional components may be needed, however, to assure high voltage transients from the terrestrial grid are not introduced into the ship while docked.

\section{FAILURE PROCESSES}

Given the withstand capabilities of the materials used for cable insulation; failure is not due to a simple overvoltage of the base insulation material. The voltages are never sufficiently high to trigger this ultimate process. Rather, there are processes that degrade the behavior. These processes are generally caused by slower charge migration processes in the material. The three dominant processes of this type that have been studied are space charge accumulation, tree formation, and void effects. All of these processes have only been observed as problems in higher voltage cables than are anticipated for future electric ships. 
However, if the insulation is reduced, higher field strengths are being produced at lower voltage and it is necessary to demonstrate that these recognized failure mechanisms do not reduce service life.

\section{A. Space Charge Accumulation}

Under a dc field, space charge can accumulate in polymeric insulation. Since the space charge changes the electric field distribution from that in the insulation when it was designed, it can cause failure. Failure from this cause typically occurs during a voltage reversal or other transient. This is due to the fact that a new voltage can be imposed across the electrodes in times much shorter than the space charge can redistribute itself, leading to high fields across portions of the insulation. The charge relaxation times depend on the specific properties of the insulator, but are expected to be in the range of minutes to hours or more [11]. It has also been determined [12] that space charge can be stored at low electric fields, because the by-products from the crosslinking process in the polymer provide ions and molecules with dipole moments that give rise to heterocharge near the electrodes when the electric field is as low as 10 $\mathrm{kV} / \mathrm{mm}$. At higher fields, charge injection from the electrodes can provide sufficient charge to convert the homocharge distribution to a heterocharge distribution. Both charge distributions distort the electric field distribution in the insulating material, so this can lead to failure.

The understanding of the role of space charge in cable failure advanced significantly with the development of acoustic methods of in-situ space charge measurement. This approach makes the correlation of space chare effects and breakdown rigorous [13].

\section{B. Tree Formation}

The term "tree" is used to describe the electric field driven growth of a potential breakdown location. This is called a "tree" because of the visual resemblance to a miniature version of a miniature winter tree growing from an electrode. The evolution of tree growth in polymeric insulation is due to the complex interaction between partial discharges and electron impact on the hollow inside of the tree [14]. It is interesting to note that this is a fundamental breakdown morphology that is common in liquids, but less likely in air presumably due to the relatively low density of air [15].

Treeing has typically been a high field phenomenon and so is not likely a serious problem for ship insulation systems. However, there is a reason to be concerned for ship applications, resulting primarily from the powering of high power pulsed loads. These loads will be used only intermittently and it may well be prudent to use undersized cables, as the required lifetime under voltage is very short compared to the life of the ship. Some experimentation will be required, however, to assure the different coefficients of thermal expansion of the various cable materials do not produce an interfacial void that can be a source of tree formation.

\section{Voids}

It is impossible to produce and maintain a void-free insulation system. The basic mechanism for voids to reduce life involved an electrical discharge within the void. A discharge requires two factors [16]. One is that the field in the void must be of sufficient magnitude to initiate a selfsustaining discharge. The other is that there must be an initiating electron available at the appropriate location. So for small enough fields and small enough voids, the inevitable voids cause no problem.

There is an additional consideration under dc. Just as space charge accumulates at the electrode interface, charges will accumulate at the void. The stability of these charges has led to applications as an acoustic transducer [17]. The accumulation of charge in the vicinity of a void raises the same potential problem that the accumulation of charge at the electrode presents - voltage changes, either steady state of transient can potentially lead to fields that are large enough to initiate damage and reduce cable life.

\section{Non-electrical factors}

Electrical factors tend to dominate failure considerations, as they are the most difficult to control. Nevertheless, the system must also be designed to survive in the chemical environment in which it operates. Since the ship is populated, the ambient environment is not expected to be a challenge, but one must also be concerned that things like spilling common cleaning materials will not destroy the insulation and damage the power system. In addition, there are mechanical issues ranging from abrasion to mechanically induced void formation. Insulation system designers are sensitive to the fact that these mechanical and chemical failures manifest themselves as electrical failures.

\section{ELECTRICAL ENVIRONMENT}

From a consideration of failure mechanisms, it is clear that the key concerns are voltage reversals and transients. These exist in all power systems, but are different in a ship than in a terrestrial power system.

\section{A. Voltage reversals}

Voltage reversals do have a benign use in dc power systems. If the time constants of the accumulation of detrimental space charge are known, periodic voltage reversals in times short compared to those time constants can minimize the space charge effects.

However, ships also provide the environment in which unattended voltage reversals can occur. To demonstrate this is indeed possible, the results of a fault study within Purdue's Reduced-Scale Naval DC Microgrid are highlighted. Figure 1 shows the Main Power Generation Module (MPGM) that is composed of a $59 \mathrm{~kW}$ wound-rotor synchronous machine followed by a rectifier and a two-stage dc-link filter. The exciter of the machine is used to control $v_{m p g m}$ to $750 \mathrm{~V}$. 
To study the influence of large transients, a lowimpedance fault was placed across the dc output terminals. The voltage $v_{m p g m}$ was measured during the fault transient and is shown in Figure 2. Therein, it can be seen that during the transient, $v_{m p g m}$ swings between positive and negative values, demonstrating a voltage reversal to the dc cable system. To prevent such a reversal, a clamping diode (shown as D2 in Figure 1) was applied. Subsequent to D2 placement, the fault study was repeated and it was shown that the voltage reversal was effectively eliminated. This illustrates that if cables are considered as part of the power electronic system design, voltage reversals may be preventable. This offers the potential to improve reliability and reduce cable size.

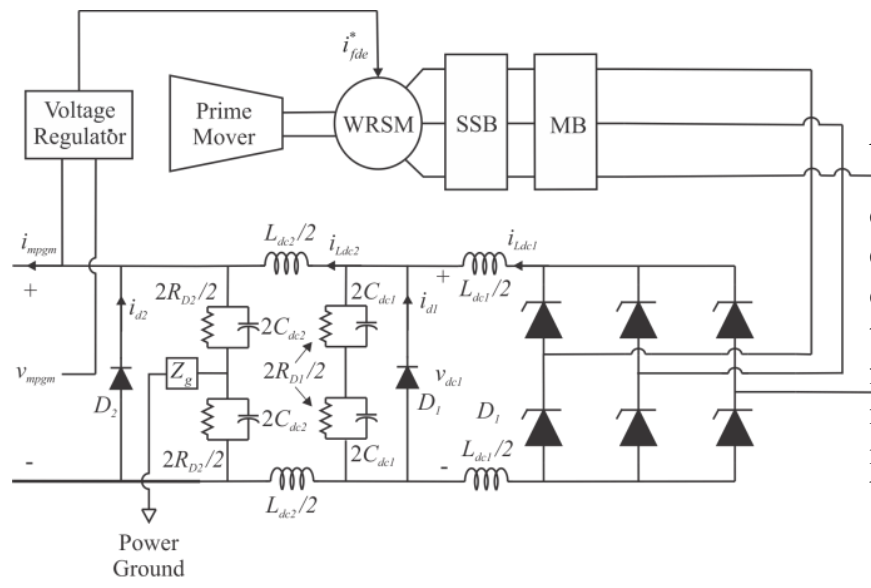

Figure 1. Main power generation module in Purdue microgrid.

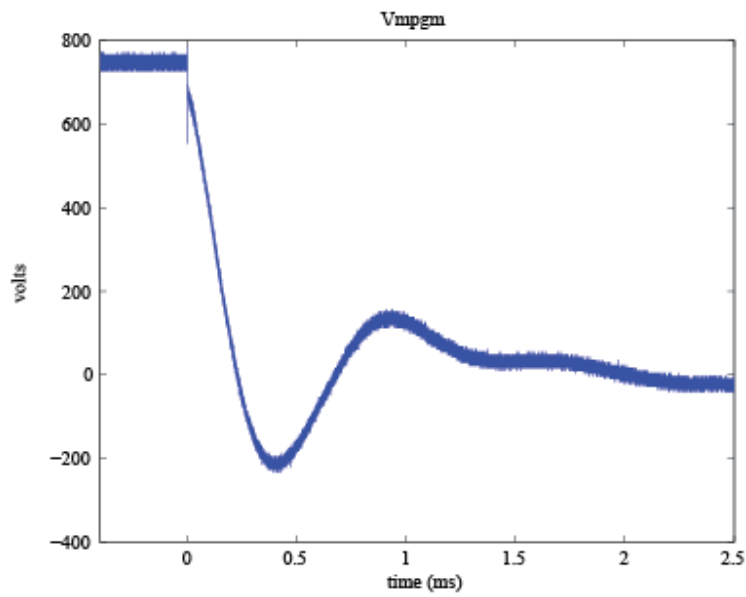

Figure 2. MPGM dc voltage during fault without cableprotecting diode.

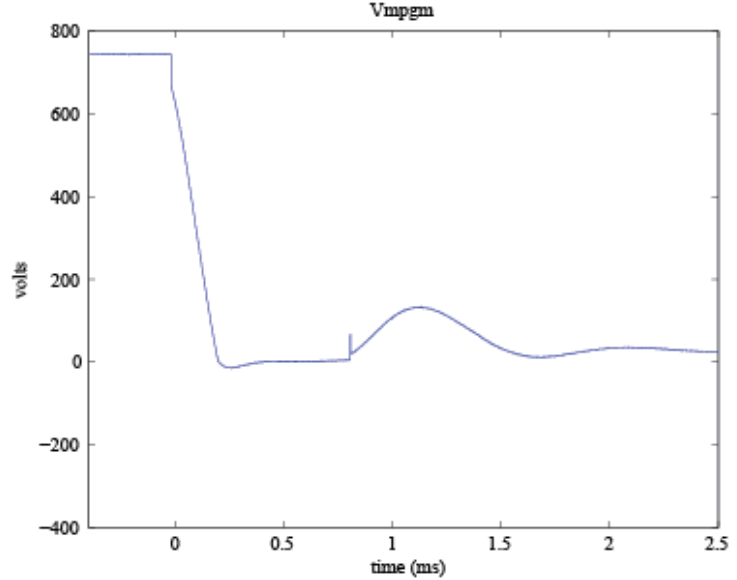

Figure 3. MPGM dc voltage during fault with cable-protecting diode.

\section{B. Transients}

A challenge for future electric ships is that they are controlled with power electronics and going forward are expected to be controlled by silicon carbide or equivalent devices that support significantly higher switching frequencies that are supported by today's silicon power electronics. The filtering or other control of these signals will be needed to minimize the over voltages induced in any component inductance. Therefore, it is likely that these transients will not be a challenge for a cable with a reduced dielectric size.

Transients that may pose a problem are those that are produced by faults in the system [17]. Providing some optimism for handling the consequences of these faults in dc systems, laboratory experiments have shown that the transients induced by faults can be smaller in dc systems than in ac systems [18]. The fundamental reason for this behavior is that when the voltage across the fault crosses through zero, as it does sixty times a second in a conventional US ship power system, the arc quickly extinguishes and reignites causing a very high rate of change of current. The current change imposed across the circuit inductance generates a large voltage spike. In a dc system, there are no voltage crossings so the arc extinguishes only as it cools, producing a much smaller rate of change of current and consequently of induced voltage.

\section{IMPLICATIONS}

With this level of analysis, variants of cable geometry like that in Figure 4 seem worthy of further examination. 


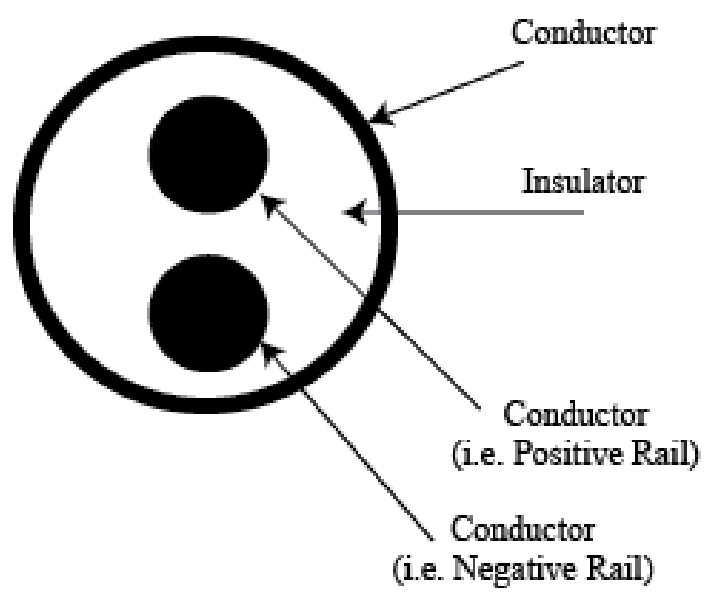

Figure 4: Typical cable cross section geometry

If installation issues can be resolved, the ideal insulator may be an epoxy with an epoxy cover on the outside of the outer "ground" conductor. If this is possible, the entire package may be closer to rectangular rather than round. The round conductors are appropriate for flexible cables, which are expected to have the same bend radius for bends in any direction. However, this approach may demand larger cables.

Also, in Figure 4, the insulation system is drawn to be much larger than it is expected to be in practice. The goal is to develop primary power cables in which the required conductor area dictates more than $90 \%$ of the cable volume, with the remainder devoted to minimizing the possibilities of electrical failure, chemical degradation, and effects of mechanical abrasion. Toward this goal, an early-stage cable design tool is being developed in which equivalent electric circuits are used to predict the electric field within the insulator under a wide range of potential geometries, material properties, and excitation levels.

\section{CONCLUSIONS}

A number of factors suggest that the cables used in future dc electric ships can be significantly smaller than those used on ac ships and in land-based operations. The reasons include:

- The lightning threat is the largest driver of insulation thickness for land-based systems, but this should be much less of a threat on ship systems.

- The primary electrical failure mechanisms in cables are well studies and are most problematic at operating voltages higher than expected on ships. Since the effects are actually electric field dependant, it is necessary to assure that going to a smaller insulation thickness does not drive these effects at lower voltage applications.

- While the cabling of a ship system is prudently designed for a 50 year service life, the cabling for high power pulsed loads will likely only be energized less than a couple of years during that fifty year span. Thus, they should be designed for intermittent rather than continuous duty, raising the possibility that they can be smaller than they would be if they were continuous duty.
- The switching transients must be controlled to protect the power electronic circuits in the ship. This transient control also raises the possibility for a more benign electrical environment for the cable than is typically assumed in cable design.

The experience base for dc power systems is more limited than for ac systems, but it is growing not only in the Navy, but in many other areas. One motivation for this trend is to reduce frequency conversions. Most electronics operate at dc, so the input power is converted from ac to dc. In addition, an increasing number of sources operate effectively at dc. So the push for simpler, more efficient systems [19] is stimulating greater adoption of dc power systems. If the cable size can be reduced, this will provide additional motivation for such systems.

\section{REFERENCES}

[1] J.H. Neher and M.H. McGrath, "The calculation of the temperature rise and load capability of cable systems," IEEE Trans. Of the American Institute of Electrical Engineers Power Apparatus and Systems, Part III, vol. 76, pp. 752-764, Oct. 1957.

[2] W.S. Zaengl, "Dielectric spectroscopy in time and frequency domain for HV power equipment. I. Theoretical considerations," IEEE Electrical Insulation Magazine, vol. 19, pp. 5-19, Oct. 2003.

[3] S. Boggs and J. Densley, "Fundamentals of partial discharge in the context of field cable testing," IEEE Electrical Insulation Magazine, vol. 16, pp. 13-18, Aug. 2002.

[4] G.C. Montanari, A. Cavallini, and F. Puletti, "A new approach to partial discharge testing of $\mathrm{HV}$ cable systems," IEEE Electrical Insulation Magazine, vol. 22, pp. 14-23, Apr. 2006.

[5] T. Tanaka, "Aging of polymeric and composite insulating materials. Aspects of interfacial performance in aging," IEEE Trans. on dielectrics and electrical insulation, vol. 9, pp. 704716, Oct. 2002.

[6] M. Ieda, "Dielectric breakdown process of polymers," IEEE Trans. on Electrical Insulation, vol. EI-15, pp. 206-224, Feb. 2007.

[7] H. Murase, S. Okabe, T. Kumai, H. Takakura, M. Takahashi, and H. Okubo, "Systematization of insulation design technology for various electric power apparatus," IEEE Trans. on dielectrics and electrical insulation, vol. 13, pp. 400-407, Apr. 2006.

[8] IEEE Standard Techniques for High-Voltage Testing, IEEE Std. 4-1995, Oct. 1995.

[9] C. Gomes and M.Z.A. Ab Kadir, "Protection of naval systems against electromagnetic effects due to lightning," Progress In Electromagnetics Research, vol. 113, pp 333-349, Nov. 1982.

[10] J.A. Plumer and J.D. Robb, "The direct effects of lightning on aircraft," IEEE Trans. on Electromagnetic Compatibility, vol. EMC-24, pp. 158-172, May 1982.

[11] S. Boggs, D.H. Damon, J. Hjerrild, J. Holboll, and M. Henriksen, "Effect of insulation properties on the field grading of solid dielectric DC cable," IEEE Trans. on Power Delivery, vol. 16, pp. 456-461, Oct. 2001.

[12] G.C. Montanari and D. Fabiani, "Evaluation of dc insulation performance based on space-charge measurements and accelerated life tests," IEEE Trans. on Dielectrics and Electrical Insulation, vol. 7, pp. 322-328, June 2000. 
[13] R. Lie, T. Takada, and N. Takasu, "Pulsed electro-acoustic method for measurement of space charge distribution in power cables under both DC and AC electric fields," Journal of Physics D: Applied Physics, vol. 26, pp. 986-993, June 1993.

[14] R.J. Densley, "An investigation into the growth of electrical trees in XLPE cable insulation," IEEE Trans. on Electrical Insulation, vol. EI-14, pp. 148-158, June 1979.

[15] B.T. Murphy, R.E. Hebner, and E.F. Kelley, "Simulating mode transitions during breakdown in liquids," IEEE Trans. on Dielectrics and Electrical Insulation, vol. 18, pp. 682-691, June 2011.

[16] P.H.F. Morshuis, J.J. Smit, "Partial discharges at DC voltage: their mechanism, detection and analysis," IEEE Trans. on Dielectrics and Electrical Insulation, vol. 12, pp. 328-340, Apr. 2005.

[17] R. Gerhard-Multhaupt, "Less can be more. Holes in polymers lead to a new paradigm of piezoelectric materials for electret transducers," IEEE Trans. on Dielectrics and Electrical Insulation, vol. 9, pp. 850-859, Oct. 2002.

[18] A. Ouroua, J. Beno, and R.E. Hebner, "Analysis of fault events in MVDC architecture," in Proc. 2009 IEEE Electric Ship Technologies Symposium, pp. 380-387.

[19] H.B. Estes, A. Kwasinski, R.E. Hebner, F.M. Uriarte, and A.L. Gattozzi, "Open series fault comparison in AC and DC microgrid architectures," in Proc. 2011 IEEE $33^{\text {rd }}$ International Telecommunications Energy Conf., pp. 1-6.

[20] R.K. Chauhan, B.S. Rajpurohit, R. E. Hebner, S.N. Singh, and F. M. Gonzalez-Longatt, "Voltage standardization of DC distribution system for residential buildings," in Proc. $20144^{\text {th }}$ International Conf. on Power and Energy Systems. 